\title{
Hayame Hussein
}

Université du Canal de Suez \& Université de La Princesse Nourah, Arabie Saoudite http://dx.doi.org/10.18778/8088-896-8.04

\section{ANALYSE DE L'ETHOS COLLECTIF DES FRANÇAIS DE CONFESSION MUSULMANE DANS LA PRESSE NUMÉRIQUE ET LES RÉSEAUX SOCIAUX}

\section{Introduction}

En 2015, Paris a été témoin d'une série d'attentats perpétrés au nom de l'Islam par des terroristes musulmans. La plupart des Français de confession musulmane ont condamné ces actes terroristes. Pourtant, beaucoup d'actes anti-musulmans ont été recensés après ces attentats ; ce qui a poussé les musulmans à exprimer, dans les courriers de lecteurs, les forums et les réseaux sociaux, leur contestation des identifications externes qui font un amalgame entre musulman et terroriste.

\section{Problématique}

Notre étude est née d'une suite de questions : quelle image les musulmans projettent-ils dans les médias en France ? A quoi est dû cet ethos hétéro-attribué ? Comment les Français de confession musulmane réagissent-ils vis-à-vis de cet ethos souvent négatif ? Pour répondre à ces questions, nous analyserons la réaction des internautes musulmans à l'égard de l'ethos collectif négatif attribué aux musulmans. Nous trouvons que cette analyse constitue un terrain d'enquête intéressant car peu d'études lui ont été consacrées, notamment dans une perspective d'analyse du discours et de l'argumentation.

L'objectif de cette étude est donc d'analyser la construction discursive de l'identité collective de la minorité musulmane en France, telle qu'elle se réalise dans les discours construits aux médias.

Notre corpus se compose d'un ensemble de textes numériques (forums, blogs, pages web, réseaux sociaux, etc.). Pour schématiser, les textes du corpus peuvent être classés en 3 sous-corpus :

Corpus presse et médias (2011-2016) : articles parus dans la presse française comme Marianne, Le Figaro, Le Monde, etc. 
Corpus blogues : textes tirés des pages personnelles et des blogues ;

Corpus d'activistes : textes extraits des pages d'organisations luttant contre l'islamophobie comme la CCIF (Collectif contre l'islamophobie en France).

Notre analyse se déroulera sur trois étapes : dans un premier temps, nous présenterons l'impact des attentats de Paris sur la minorité musulmane française. Ensuite, nous étudierons l'ethos hétéro-attribué des musulmans tel qu'il se construit dans la presse de l'extrême droite et celle de la gauche ainsi que dans les réseaux sociaux (facebook et twitter). Puis, nous analyserons la réaction des internautes musulmans vis-à-vis de cet ethos, souvent négatif ; ce en illustrant leur ethos collectif auto-attribué tel qu'ils projettent dans leurs débats et leurs commentaires. De même, nous mettrons l'accent sur leur tentative de retravailler cet ethos. Enfin, nous étudierons les arguments qu'ils avancent à leurs compatriotes pour retravailler cet ethos prédiscursif négatif.

Nous nous baserons dans notre étude sur les travaux de Chaïm Perelman, de Ruth Amossy et d'Eithan Orkibi dans le domaine de l'argumentation et de l'analyse de discours.

\section{L'impact des attentats de Paris sur les Français de confession musulmane}

Selon l'Observatoire national contre l'islamophobie, « pour la période du $1^{\mathrm{er}}$ janvier au 31 décembre 2015, il a été enregistré 429 actes antimusulmans, contre 133 pour l'année 2014, soit 223\% d'augmentation $\gg^{1}$. L'Observatoire national contre l'islamophobie trouve un lien direct entre l'augmentation de ces actes et les attentats djihadistes de janvier 2015. Ces actes islamophobes prennent plusieurs formes :

- Violences verbales et physiques ;

- Discriminations à l'entrée d'un restaurant ou d'une salle de sport ;

- Dégradation de lieux privés ;

- Profanation de cimetières ou harcèlement de jeunes lycéennes portant des jupes longues ;

- Discrimination contre musulmans à l'embauche.

Dans les médias, les propos islamophobes se multiplient ; prenons à titre d'exemple, les titres des articles parus dans le journal numérique Riposte Laïque après les attentats de Charlie Hebdo :

- «Pour gagner cette guerre, il faudra éradiquer l'islam en France $»^{2}$.

1 A. Zekri, Communiqué: Bilan des actes antimusulmans du $1^{\text {er }}$ janvier au 31 décembre 2015, www.lecfcm.fr (page consultée le 4 mai 2016).

2 Cyrano, Riposte Laïque, www. ripostelaique.com (page consultée le 4 mai 2016). 
- «Soit nous nous débarrassons de l'islam, soit l'islam se débarrasse de nous $\gg^{3}$.

- «Seule l'expulsion des musulmans mettra fin aux attentats musulmans $\gg^{4}$.

- Ces propos islamophobes nous poussent à étudier l'ethos hétéro-attribué des musulmans tel qu'il se construit dans la presse d'extrême droite.

\section{L'ethos hétéro-attribué de la minorité musulmane française}

D’après Amossy, l'ethos est défini dans la rhétorique classique comme « l'image de soi que le locuteur construit à travers son discours pour exercer une influence sur son auditoire $\gg^{5}$. Cette image est aussi basée sur la prise en compte de son ethos prédiscursif « qui, souligne Amossy, catégorise le locuteur, sa réputation individuelle, l'image de sa personne qui dérive d'une histoire conversationnelle ou textuelle, son statut institutionnel et social $\gg^{6}$.

Quant à la notion d'ethos collectif, elle renvoie, selon Orkibi, « à l'image de soi d'un groupe : une image mobilisée ou reflétée dans la parole de l'individu appartenant à ce groupe [...] mais aussi une image de soi collective qui est exprimée par un groupe, à savoir l'ethos du locuteur $\gg^{7}$. L'ethos collectif est renforcé « sur la base de la race, de l'âge, du sexe (gender) ou du statut professionnel, et consiste en l'élaboration de traits caractéristiques propres au groupe, à savoir l'apparence, le langage, les valeurs et les croyances, les symboles visuels $\gg^{8}$.

Dans le cadre de notre étude, nous trouvons important d'intégrer la notion élaborée par Charaudeau d'un ethos collectif hétéro-attribué, qui correspond à la projection sur un groupe d'une image sociale identitaire de la part de ceux qui ne lui appartiennent pas. Cet ethos collectif est fondé sur les aprioris et les stéréotypes attachés à ce groupe :

3 M. Tonarelli, Riposte Lä̈que, www.ripostelaique.com/soit-nous-nous-debarrassons-de-lislam-soit-lislam-se-debarrasse-de-nous.html (page consultée le 10 mai 2016). 2016).

4 M. Lépante, Riposte Laïque, www.ripostelaique.com (page consultée le $1^{\text {er }}$ mai

5 R. Amossy, «Ethos », [in] P. Charaudeau et D. Maingueneau (éds.), Dictionnaire d'analyse du discours, Paris, Seuil, 2002, p. 239.

6 R. Amossy, La présentation de soi. Ethos et identité verbale, Paris, PUF, 2010, p. 73.

7 E. Orkibi, Les étudiants de France et la guerre d'Algérie. Identité et expression collective de l'UNEF (1954-1962), Paris, Syllepse, 2012, p. 22.

8 E. Orkibi, « Ethos collectif et Rhétorique de polarisation : le discours des étudiants en France pendant la guerre d'Algérie $\gg$, Argumentation et Analyse du Discours, mis en ligne le 21 septembre 2008, www.aad.revues.org/438 (page consultée le 20 mai 2015). 
Les individus, du fait de leur appartenance à un groupe, partagent avec les autres membres du groupe des caractères similaires, ce qui donne l'impression, vu de l'extérieur, que ce groupe représente une entité homogène. Une fois de plus, il est essentialisé par un regard extérieur, ce qui engendre des stéréotypes [...] L'ethos collectif correspond à une vision globale, mais à la différence de l'ethos singulier, il n'est construit que par attribution apriorique, attribution d'une identité émanant d'une opinion collective vis-à-vis d'un groupe autre'.

Le stéréotype contribue donc à la construction de ce qu'Amossy appelle la « mémoire collective $»^{10}$; ainsi le stéréotype joue-t-il un rôle important dans la compréhension réciproque et l'identification collective.

Dans une recherche élaborée par Harris Interactive pour l'Institut Montaigne ${ }^{11}$, on a démontré que la discrimination dont souffrent les musulmans sur le marché du travail est due aux stéréotypes négatifs qui leur sont associés. En fait, « lorsqu'on demande aux Français de préciser les mots ou expressions qui leur viennent spontanément à l'esprit quand ils pensent à la religion musulmane, deux familles de stéréotypes émergent, tous négatifs $\gg^{12}$. La première famille fait référence à une pratique extrémiste de la religion : «intégrisme », « intolérance », « fanatisme ». Les musulmans y apparaissent surtout comme des personnes agressives, violentes et menaçantes qui attisent les conflits. La deuxième famille de stéréotypes est constituée des termes «femme » et « voile ». Elle suggère que les Français assimilent l'islam à une religion qui ne traite pas les hommes et les femmes sur un pied d'égalité.

Dans notre étude, nous avons remarqué que les écrits médiatiques sont très marqués par les mêmes stéréotypes cités dans l'étude de l'Institut Montaigne ; prenons, à titre d'exemple, cette déclaration d'un blogueur sur Twitter où il affirme : «Si tous les musulmans ne sont pas des terroristes tous les terroristes sont musulmans $\gg^{13}$. La citation du blogueur relève de la première famille de stéréotypes où les termes islamiste ou terroriste sont associés directement à l'islam. Il s'agit d'une argumentation fondée sur un sophisme de généralisation où, à partir d'un seul ou quelques cas particuliers, on généralise, sans avoir analysé

9 P. Charaudeau, Le discours politique. Les masques du pouvoir, Paris, Vuibert, 2005, p. 90.

10 R. Amossy, La présentation de soi..., p. 48.

11 Cf. Harris Interactive pour l'Institut Montaigne, « Le regard des Français sur la religion musulmane », LCP et Tilder, 2013, www.institutmontaigne.org (page consultée le 10 février 2016).

12 Ibidem.

13 B. Kilmeadem, Twitter, www.mobile.twitter.com/Navymat/ (page consultée le 20 février 2016). 
l'ensemble des cas ou à défaut un échantillonnage représentatif ${ }^{14}$. En général, plusieurs clichés se répandent dans les médias, nous pouvons en citer :

- Le musulman est violent et menaçant ;

- L'Islam est incompatible avec la République ;

- Le musulman est arriéré et rejette les valeurs occidentales ;

- Le musulman est un envahisseur, en contradiction avec l'identité française.

Ces clichés sont répétés dans les débats des internautes français en réaction aux attentats du 13 novembre 2015, prenons pour exemple cette citation de Siegfried Däbritz, l'un des organisateurs du mouvement xénophobe Pegida (Patriotes Européens Contre l'Islamisation des Pays d'Europe), parue dans Le Figaro : « Celui qui ne fait pas le lien entre l'islam, les réfugiés et les attentats de Paris est un idiot $\gg{ }^{15}$. Observons également la citation de Salem Ben Ammar, un blogueur français : «Un musulman modéré est un djihadiste en sommeil qui attend son heure pour passer à l'action [...] L'islam modéré est un leurre comme si un fasciste pouvait avoir le sens de la mesure $\gg^{16}$. Dans cette citation, pour exprimer sa position hostile aux musulmans, le blogueur a recours à trois procédés argumentatifs : la définition argumentative ${ }^{17}$, l'ironie et la généralisation. La définition argumentative consiste, selon Plantin, «à définir un terme de telle sorte que la définition exprime une prise de position, favorable ou défavorable, vis-à-vis de l'objet défini $\gg^{18}$. Lorsqu'une prise de position est exprimée, celle-ci redéfinit argumentativement le terme auquel elle se réfère. Dans notre exemple, le blogueur redéfinit le musulman modéré de façon ironique pour nier indirectement son existence puisque le fait d'attendre l'heure de passer à l'action contredit toute modération. La définition, dans ce cas-ci, joue en quelque sorte le rôle d'une thèse que le blogueur cherche à défendre, à appuyer et à étayer.

Quant à la deuxième famille de stéréotypes, elle renvoie à la soumission de la femme. Selon ce stéréotype, la musulmane est opprimée, malheureuse, inapte à toute activité sociale et obligée de porter le voile. Prenons ce commentaire d'un internaute français : « L'islam actuel ne sépare pas la foi du pouvoir sur les

14 Cf. M. S. John, Système de logique déductive et inductive, (Système de Logique. Livre 5. Les sophismes), Bruxelles, Pierre Mardaga, 1988, p. 357.

15 Le Figaro, le 17 novembre 2015, www.pressreader.com/france/le-figaro (page consultée le 20 février 2016).

16 S. B. Ammar, www.salembenammar.wordpress.com (page consultée le 15 février 2016).

17 Dans la définition argumentative, en définissant un terme, le locuteur exprime aussi une prise de position qui peut être favorable ou défavorable vis-à-vis de l'objet défini.

18 C. Plantin, L'argumentation, Paris, Éditions du Seuil, 1996, p. 53-54. 
hommes et considère les femmes comme inférieures aux hommes. [...]. La loi de 1905 doit donc évoluer pour imposer aux religions de respecter les principes de la République. Sinon, ce sont des sectes et doivent être interdites $\gg^{19}$.

\section{Stéréotype et allusion historique}

Le stéréotype du musulman violent et sanguinaire a parfois pour origine une allusion historique. En fait, le discours de droite en France en ce qui concerne les musulmans se caractérise par le recours récurrent à l'allusion historique pour déjouer la vigilance de la censure. Prenons, à titre d'exemple, cette allusion historique parue dans le Nouvel Observateur où le journaliste Ramses Kefi, tout en abordant la notion de « musulman modéré », a mis comme illustration ce document :

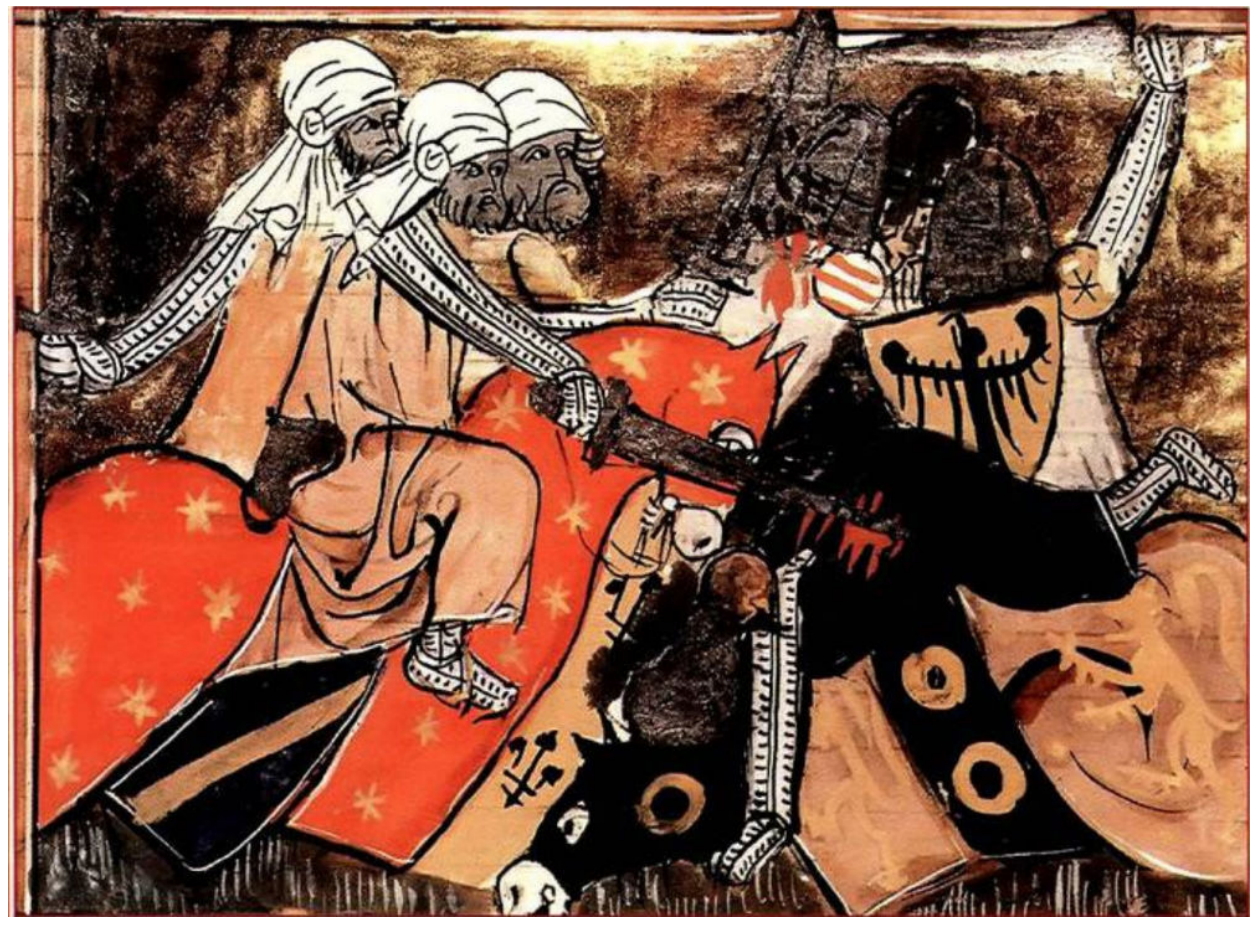

1. Bataille entre croisés et chevaliers arabes, France, XIV siècle, source : Le Nouvel Observateur, 6/5/2015

19 Tony, Le point, le 19/11/2015, www.lepoint.fr (page consultée le 10 février 2016). 
Le document, accompagné de la légende «Bataille entre croisés et chevaliers arabes, France, $\mathrm{XIV}^{\mathrm{e}}$ siècle $\gg$, fait allusion au conflit héréditaire entre le Christianisme et l'Islam. Selon Amossy, « l'allusion historique [...] présente à la surface de l'énoncé des marques [...] qui permettent d'activer un texte-source, un arrière-fond textuel qui fait partie du répertoire dont dispose le public $\gg^{20}$. Les éléments familiers auxquels renvoie l'allusion sont en l'occurrence ceux d'une doxa sur l'image des musulmans qui étaient, à une certaine époque de l'histoire, perçus comme des conquérants violents.

Après avoir montré l'impact des stéréotypes anti-musulmans sur le discours médiatique en France, nous étudierons comment les musulmans tentent de retravailler l'ethos négatif qu'on leur attribue dans les médias.

\section{Retravail de l'ethos préalable négatif}

Le retravail de l'ethos préalable constitue, selon Amossy, « la pratique discursive au gré de laquelle le locuteur investit son image préexistante pour construire un ethos discursif plus conforme à son projet argumentatif $\gg^{21}$. C'est grâce à l'ethos discursif que le locuteur choisit de modifier ou de confirmer certains éléments de l'ethos préalable. L'ethos discursif correspond à la notion d'ethos rhétorique élaborée par Aristote : « on persuade par le caractère quand le discours est de nature à rendre l'orateur digne de foi [...] Mais il faut que cette confiance soit l'effet du discours, non d'une prévention sur le caractère de l'orateur $\gg^{22}$. Declercq souligne ce point :

Tout ce qui, dans l'énonciation discursive, contribue à émettre une image de l'orateur à destination de l'auditoire. Ton de voix, débit de la parole, choix des mots et arguments, gestes, mimiques, regard, posture, parure, etc., sont autant de signes, élocutoires et oratoires, vestimentaires et symboliques, par lesquels l'orateur donne de lui-même une image psychologique et sociologique ${ }^{23}$.

L'ethos d'un discours résulte donc d'une interaction entre trois facteurs :

- l'ethos prédiscursif (préalable);

- l'ethos dit où l'énonciateur donne des informations sur sa propre personne dans le contenu de son discours ;

${ }^{20} \mathrm{R}$. Amossy, « Israël et les juifs dans l'argumentation de l'extrême droite : doxa et implicite », Mots, n 58, mars 1999 (Argumentations d'extrême-droite), p. 84.

${ }^{21}$ R. Amossy, «Ethos »..., p. 239.

22 Aristote, Rhétorique (trad. P. Chiron), Paris, Flammarion, 2007, Livre premier, chapitre II, 1356 a, p. 126.

23 G. Declerc, L'art d'argumenter. Structures rhétoriques et littéraires, Paris, Éditions Universitaires, 1993, p. 48. 
- l'ethos montré qui se révèle implicitement dans l'acte d'énonciation, il ne se dit pas dans l'énoncé. Il reste par nature au second plan de l'énonciation : il est perçu et ne fait pas l'objet du discours.

L'étude du re-travail de l'ethos préalable d'un groupe exige d'analyser la manière dont ce groupe élabore sa propre image et « retravaille, selon Orkibi, une série de représentations sociales préétablies $\gg^{24}$. Nous étudierons notamment l'image que les internautes musulmans ont construite en parlant d'eux-mêmes explicitement (ethos dit) et implicitement (ethos montré).

En étudiant les débats dans les réseaux sociaux après les attentats de Paris, nous avons trouvé que les musulmans tenaient notamment à affirmer leur identité française. L'ethos projeté est plutôt dit, prenons, à titre d'exemple, cette citation :

En pleine crise d'identité, me voilà plus rassuré. Français de nationalité, profondément loyal à ma patrie et lä̈que dans ma profession. Je reste musulman d'occident modéré et militant non violent mais certes radical pour la défense des droits de l'Homme universels ${ }^{25}$.

Le locuteur déclare explicitement être français, loyal, laïque, musulman modéré, militant non violent, etc.

Voilà également la déclaration que Jamel Debbouze a faite au journal Sept à Huit sur un ton grave et meurtrier, selon l'expression du journaliste :

J'ai passé mon temps à ne pas dire que j'étais musulman. Pas parce que je n'en étais pas fier, loin de là, parce que je considérais que ce n'était pas un sujet, qu'on n'avait pas besoin de revendiquer son identité, sa différence [...] Aujourd'hui, j'ai presque besoin de le revendiquer, comme pour dire 'ne vous inquiétez pas, on est pareils'. Je suis français, musulman, artiste. Je suis né à Barbès, j'ai grandi à Trappes. Je suis père de 2 enfants, marié à une chrétienne, journaliste, très très belle. Et ça pour moi, c'est la France ${ }^{26}$.

L'ethos dans les propos de Debbouze est à la fois dit et montré. L'ethos est dit lorsqu'il liste un à un ses traits d'appartenance identitaire en les organisant selon une certaine priorité : Je suis français, musulman, artiste, etc. De même, l'ethos est montré lorsque les propos de Debbouze renvoient l'ethos d'un musulman tolérant qui accepte la différence, rejetant ainsi le stéréotype de l'incompatibilité entre l'islam et les valeurs de la République.

${ }^{24}$ E. Orkibi, «Ethos collectif... », p. 4.

${ }^{25}$ Douaisien59, « Qui sont les musulmans modérés ? 》, www.tariqramadan.com/ qui-sont-les-musulmans-moderes (page consultée le $1^{\text {er }}$ janvier 2016).

${ }^{26}$ Diply, www.diply.com (page consultée le 5 janvier 2016). 
Nous trouvons que le recours des locuteurs à l'ethos dit dans les deux derniers exemples peut être justifié par le souci de retravailler l'image négative du musulman, dressée par les terroristes en citant clairement les caractéristiques du vrai musulman dont ils projettent l'image.

Au niveau des acteurs sociaux, la CCIF (Le Collectif Contre l'Islamophobie en France) a mené une campagne visant à déconstruire les clichés anti-musulmans. La campagne était signée : « Nous sommes la Nation », elle avait pour but de

renforcer l'appartenance des citoyens musulmans à la communauté française et l'importance des valeurs énoncées dans le devise nationale. Les français musulmans font eux aussi partie de la Nation, par naissance, mais aussi par leur sentiment d'appartenance, par leur contribution quotidienne et historique à la vie du pays ${ }^{27}$.

La campagne a choisi comme slogan cette image symbolique :

\section{Nous aussi sommes la Nation}

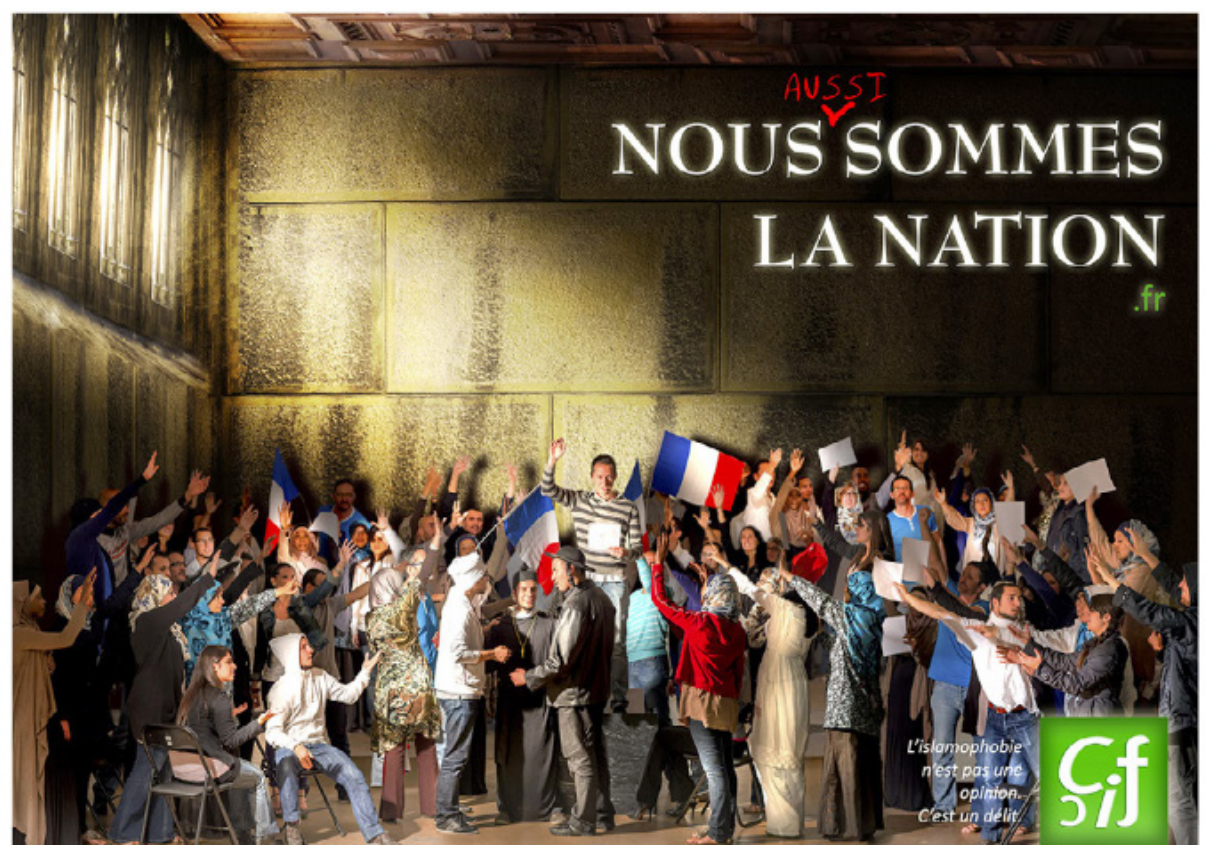

2. Source : www.islamophobie.net

27 S. Rahmani, Nous sommes la nation, www.islamophobie.net (page consultée le 6 janvier 2016). 
L'affiche constitue une interprétation artistique libre du tableau « Le Serment du Jeu de Paume » de David. L'affiche montre une foule de citoyens se saisissant du drapeau, qui sont réunis avec le respect des différences de chacun et visent à restaurer les valeurs fondatrices de la République. Dans cette foule, les musulmans français revendiquent leur pleine appartenance à la Nation, la pleine compatibilité de leur citoyenneté et de leur pratique religieuse.

L'ethos dans cette campagne est à la fois dit et montré:

- Dit : Nous = la nation

- Montré : respect de la laïcité et tolérance.

Observons également le choix du pronom personnel 'nous' qui résulte de l'association de 'je' et 'vous' et donne lieu à un mécanisme d'inclusion des musulmans dans l'espace public.

\section{Ethos et choix des arguments}

Par ailleurs, l'ethos montré que projettent les internautes musulmans est élaboré par le moyen du choix des arguments et des procédés qu'ils utilisent pour se défendre et en même temps pour retravailler leur ethos négatif. Parmi ces procédés, nous pouvons citer l'argumentation par les caricatures. Observons, par exemple, cette caricature qui nie toute relation entre l'islam et les actes terroristes :

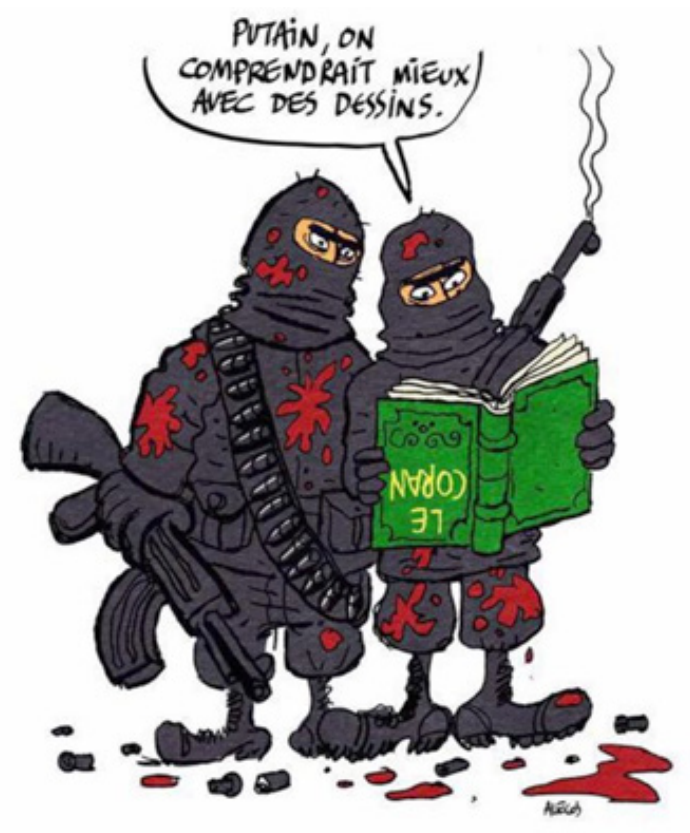

3. Source : www.twitter.com/rakizama 
La caricature repose sur ce syllogisme :

Prémisse 1 : pour représenter l'islam, il faut comprendre le Coran (le livre Saint des musulmans);

Prémisse 2 : or les terroristes ne savent même pas lire le Coran ;

Conclusion : donc, les terroristes ne représentent pas l'Islam.

La caricature constitue une forme de l'argumentation par l'illustration dont le rôle est, selon Perelman, de « renforcer l'adhésion à une règle connue et admise, en fournissant des cas particuliers qui éclairent l'énoncé général, lesquels montrent l'intérêt de celui-ci par la variété des applications possibles et augmentent sa présence dans la conscience $\gg^{28}$.

Un autre internaute diffuse cette illustration pour dénoncer l'amalgame entre musulman et terroriste :

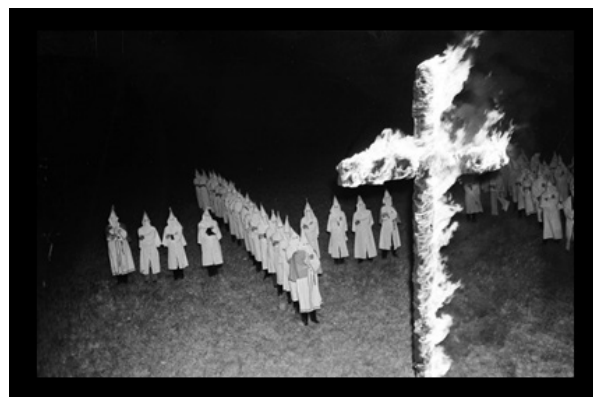

Personne n'a jamais considéré ces gens comme représentatifs des chrétiens

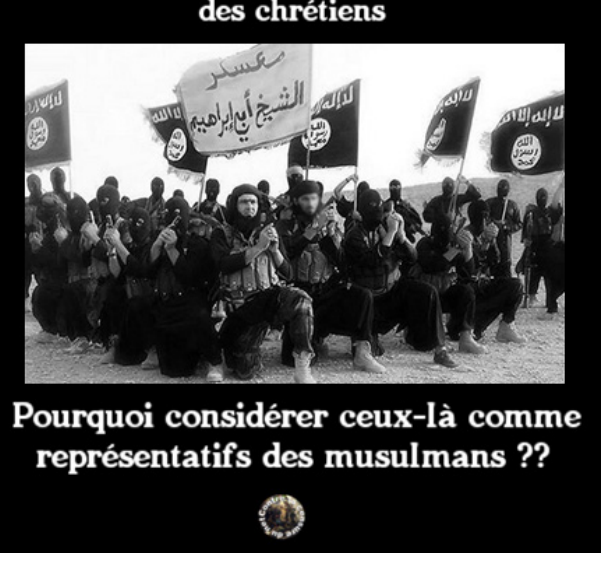

4. Source : www.twitter.com/majden3

${ }^{28}$ Ch. Perelman, Traité de l'argumentation : la nouvelle rhétorique, Bruxelles, Éditions de l'Université de Bruxelles, 1958, p. 471. 
L'internaute ajoute : « Les Français ne sont pas tous chrétiens, les musulmans ne sont pas tous des terroristes. Stop à l'amalgame $\gg^{29}$.

L'illustration est formée de deux parties : dans la première, apparaissent les adeptes d'une secte extrémiste chrétienne qui sont en train de pratiquer des rites violents. Sous l'image est écrit : « Personne n'a jamais considéré ces gens comme représentatifs des chrétiens $\gg$. Sur la deuxième partie de l'illustration, nous voyons les daëchiens en uniforme de guerre, qui sont en train de prier de façon extravagante ; et sous la photo est écrit : «Pourquoi considérer ceux-là comme représentatifs des musulmans ? » L'internaute réfute l'amalgame en ayant recours à l'argument de deux poids deux mesures qui signifie : juger deux choses analogues avec partialité, selon des règles différentes. Dans notre illustration, le même cas d'extrémisme est jugé différemment selon les pratiquants. L'argumentation de l'internaute repose sur l'argument par la comparaison qui établit un parallèle entre deux situations : l'extrémisme chrétien et l'extrémisme musulman. Il montre que les deux situations doivent être considérées et traitées de la même façon.

Il existe également un autre type d'argumentation dont l'usage est très récurrent dans les réseaux sociaux, à savoir l'argument d'autorité qui consiste à faire admettre une thèse en la rapportant à son auteur, considéré comme source d'autorité et digne de foi. C'est le cas dans cette affiche qu'un internaute a photographiée et publiée pour prouver que le Coran interdit l'assassinat d'autrui :

\section{Violence}

Celui qui a tué un homme qui n’a commis aucune violence sur terre, ni tué, c'est comme s'il avait tué tous les hommes. Celui qui sauve un seul innocent, c'est comme sil avait sauvé l'humanité tout entière...

(Cons, V, 32)

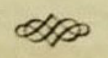

Ne tuez pas la personne humaine, car Allah l'a déclarée sacrée.

(Conan, VI. 15t)

${ }^{29}$ Majden3, Twitter, www.twitter.com/majden3. 


\section{Conclusion}

Dans le cadre de notre étude, nous avons remarqué que les écrits médiatiques concernant la population musulmane de France sont très marqués par des stéréotypes racistes qui font l'amalgame entre l'islam et l'intégrisme. En étudiant les débats dans les réseaux sociaux après les attentats de Paris, nous avons trouvé que les musulmans tenaient notamment à revendiquer leur identité française. L'ethos projeté est en premier lieu dit. Quant à l'ethos montré que projettent les internautes musulmans, il est élaboré par le moyen du choix des pronoms (nous d'inclusion), celui des arguments et des procédés qu'ils utilisent pour retravailler leur ethos négatif. De même, nous avons conclu que la caricature constitue l'un des procédés argumentatifs les plus efficaces dans le re-travail de l'ethos.

Le champ d'étude de l'ethos projeté dans les médias est très vaste, et dépasse le cadre limité d'un article scientifique ; nous avons dû omettre beaucoup d'exemples significatifs pour respecter la longueur de l'article. Notre article peut être considéré comme une amorce à une étude plus vaste où seront également analysées les conversations entre les internautes : soit entre musulmans et non musulmans, soit entre musulmans modérés et musulmans fanatiques.

Nous pensons en outre qu'il est devenu impératif de faire des études sociologiques et linguistiques sur les procédés argumentatifs par lesquels les discours des djihadistes arrivent à recruter de nouveaux membres de Daëch, notamment à travers les conversations sur les réseaux sociaux qui constituent un premier outil de recrutement des daëchiens.

\section{Analysis of the collective ethos of French Muslims in the digital press and social network}

After Paris attacks in 2015, the image projected by the Muslim minority is very negative. The objective of this study is to analyze the discursive construction of the collective ethos of the Muslim minority in France, through the speeches in media. Our corpus consists of a group of digital texts (articles, blogs, forums, social media, etc.) Our search is at the crossroads of two disciplines: argumentation and discourse analysis. Our analysis proceeds in four steps: first, we present the impact of Paris attacks on the Muslim minority. Then, we study the preliminary ethos of Muslims as it is constructed in the media. Afterwards, we analyze the collective ethos self-attributed, presented by Muslims in response to their negative ethos. Finally, we study the arguments they advance to rework this negative preliminary ethos.

Keywords: Muslim minority - attacks in Paris - argumentation - discourse analysis - ethos Mots-clés : minorité musulmane - attentats de Paris - argumentation - analyse du discours - ethos 